\title{
Análisis sociolingüístico de las diferencias de género en los patrones narrativos de historias de experiencia personal en el habla juvenil de Santiago de Chile*
}

\author{
Silvana Guerrero González ${ }^{* *}$ \\ Universidad de Chile
}

\begin{abstract}
Resumen
En la presente investigación se da cuenta de las diferencias de género en el empleo de patrones narrativos (Reyes 1996) en las historias de experiencia personal de jóvenes de Santiago de Chile. El objetivo principal de la indagación fue identificar tales diferencias y establecer su incidencia en el desarrollo de las narraciones. Tras el análisis de 64 relatos, se concluyó que prácticamente no existen diferencias de género en el uso de los patrones narrativos de los relatos efectuados por hombres y por mujeres. Sin embargo, se detectó una diferencia a nivel de estrato social en relación con la preferencia del patrón clásico de relato de las historias empleado, sobre todo, por los hablantes de los niveles inferiores de la escala de estratificación social. Sobre la
\end{abstract}

* En este artículo se sintetizan los resultados más relevantes de una de las secciones de nuestra tesis de Magíster en Lingüística con mención en Lengua Española por la Universidad de Chile, "Análisis sociolingüístico de las diferencias de género en narraciones de experiencias personales en el habla juvenil de Santiago de Chile", dirigida por el Dr. Luis Prieto Vera.

** Para correspondencia, dirigirse a: Silvana Guerrero González (siguerrero@u.uchile.cl), Universidad de Chile, Facultad de Filosofía y Humanidades, Departamento de Lingüística, Av. Cap. Ignacio Carrera Pinto 1025, Ñuñoa, Santiago, Chile. 
base de los resultados del estudio, se propone una explicación inicial de tal diferencia mediante el concepto de "oralidad primaria".

Palabras clave: narraciones de experiencia personal, habla juvenil, patrones narrativos, oralidad primaria.

A SOCIOLINGUISTIC ANALYSIS OF GENDER DIFFERENCES IN NARRATIVE PATTERNS USED IN THE ORAL RECOUNTING OF PERSONAL EXPERIENCE IN THE SPEECH OF YOUNG SPEAKERS FROM SANTIAGO, ChILE

Abstract

This research study aims at accounting for gender differences in the use of narrative patterns (Reyes 1996) by young adult subjects from Santiago of Chile when narrating their personal experiences. The main objective was to identify such differences and establish their frequency of occurrence in the development of the narrations. After analyzing 64 narratives, we concluded that there were practically no differences in the subjects' use of narrative patterns. However, an unexpected preference was found for the use of the standard narrative pattern by the subjects representing the lower levels of the social scale: the frequency of occurrence of such pattern in their narratives was higher than that found the other levels. On the basis of these results, we propose an initial explanation of such difference through the concept of "primary orality".

Key words: narratives of personal experience, chilean youths' speech, narrative patterns, primary orality.

Recibido: 15/07/11. Aceptado: 12/10/11.

\section{INTRODUCCIÓN}

El interés por el análisis del discurso oral se ha acrecentado de manera notable en los últimos años (De Beaugrande 2000; van Dijk, 2005 y Charaudeau y Maingueneau 2005). En lo que respecta a la relación existente entre género y discurso, esta encuentra un sostenido y creciente desarrollo a partir de la década de 1960. De esta forma, se ha generado un sinnúmero de trabajos abocados a indagar en dicha relación, sobre todo, en el contexto de la 
lingüística norteamericana (Lakoff 1973; Jones 1980; Tannen 1990a, 1990b, 1996 y Coates 2009), situación que no ha alcanzado un nivel comparable en el ámbito hispánico. Al respecto, Soler (2004) señala que los trabajos existentes sobre la variación discursiva en español son escasos, pues centrarse en ellos implica dilucidar si en una determinada situación, algunas características socio-demográficas de los usuarios del lenguaje, como el sexo-género, el nivel etario o el estrato social, pueden construirse y ser relevantes en el discurso, o pueden ser menos relevantes o incluso irrelevantes en ese mismo contexto. A partir de lo anterior, esta investigación se perfila como una contribución a la descripción sociolingüística del español aplicado a la lengua española, en general, y al hablado en Santiago de Chile, en particular.

El objetivo principal del estudio es analizar las diferencias de género en el empleo de los patrones narrativos en un subtipo específico del discurso narrativo juvenil en Santiago de Chile, a saber, en las narraciones de experiencia personal. Para tal propósito, se empleará como matriz analítica la propuesta de Reyes (1996) y se realizará un estudio cuantitativo, exploratorio aplicado a una muestra de entrevistas sociolingüísticas. En este estudio se sustenta la hipótesis de la existencia de diferencias de género en el empleo de patrones narrativos en el discurso narrativo juvenil. Nuestra indagación deberá verificar si existen o no tales diferencias, analizando, aquellas que se encuentren, a la luz de las categorizaciones pragmático-discursivas que las justifiquen de modo significativo. Cabe destacar que se ha seleccionado el habla del segmento etario juvenil de la población debido a que, primero, se ha considerado que, "a diferencia de otras variables sociales, la edad es la más objetiva e inmutable de todas" (Chambers 2003:146) y, segundo, porque se intenta dar cuenta de los rasgos peculiares y distintivos de los relatos juveniles, a fin de contrastarlos en futuras investigaciones con los de otros grupos etarios de la comunidad en estudio.

\title{
2. MARCO CONCEPTUAL
}

\author{
2.1. El Discurso NARRATIVO Y LA PROPUESTA DE LABOV \\ Y WALETZKY (1967) Y LABOV (1972) EN TORNO A \\ LAS NARRACIONES DE EXPERIENCIA PERSONAL
}

A partir de la concepción de los componentes (o niveles) del texto de Werlich (1975) y del concepto de superestructura de van Dijk (2000), Adam (1999) plantea secuencias discursivas prototípicas, que responden 
a tipos relativamente estables de combinación de enunciados, organizados mediante su estructura jerárquica interna (esquema) y por su unidad compositiva (plan). Este autor señala que se trata de unidades modélicas, sintetizadas en cinco tipos de secuencias: 1) narrativa, 2) descriptiva, 3) argumentativa, 4) explicativa y 5) dialogal. De acuerdo con Bassols y Torrent (2003), la narración es el tipo de secuencia discursiva que ha generado el mayor número de estudios, análisis y reflexiones. Por otra parte, según Calsamiglia y Tusón (1999), no solo se narra para contar historias, sino que las narraciones están presentes también en los diálogos, en las anécdotas, en los discursos argumentativos y en los chistes, entre otras instancias específicas de uso lingüístico. En particular, las narraciones de experiencias personales constituyen una de las formas de expresión verbal más utilizadas por las personas. Asimismo, según Moreno Fernández (1998), la narración oral es una de las estructuras (o subestructuras) textuales más interesantes y complejas que pueden incorporarse dentro de las conversaciones.

La bibliografía sobre narraciones es extensa. Sin embargo, el trabajo seminal de Labov y Waletzky (1967), así como el de Labov (1972), han demostrado ser, ampliamente, el modelo de más alto rendimiento empírico en la investigación sobre el problema, como ha quedado de manifiesto en varios estudios sobre narrativas (Reyes 1996; Soler 2004; Jiménez 2006; Acuña 2006 y Shiro 2007, por solo citar algunos ejemplos). En estricto sentido, Labov (1972: 360) define la narración como "one method of recapitulating past experience by matching a verbal sequence of clauses to the sequence of events which (it is inferred) actually occurred [un método de recapitulación de la experiencia pasada adecuando una secuencia verbal de proposiciones a la secuencia de sucesos que (se infiere(n)) ocurrieron realmente" (la traducción es nuestra)]. De esta forma, la narración es considerada como un texto que comunica una serie de eventos que ocurren en el tiempo, pero donde, a su vez, se percibe de manera subyacente una relación causal o temática. Labov y Waletzky (1967) y Labov (1972) distinguen dos tipos de relatos: los relatos mínimos, compuestos de -al menos- dos cláusulas narrativas en pasado y los relatos completos. Dichos autores plantean que una narración de experiencias personales plenamente formada exhibe seis rasgos estructurales bien definidos, cada uno con una posición característica en la narración. Los seis rasgos estructurales de una secuencia narrativa plenamente constituida son: 1) Resumen, 2) Orientación, 3) Acción de complicación de la narración, 4) Evaluación, 5) Resultado o resolución y 6) Coda o moraleja.

Cabe señalar que, para los fines de nuestra investigación, la importancia de los aportes del trabajo de Labov y Waletzky (1967) radica en que constituye una parte esencial de la propuesta de la existencia de siete patrones narrativos realizada por Reyes (1996), en la que basaremos nuestro estudio. 


\subsubsection{Los patrones narrativos}

A partir del análisis narrativo realizado por Labov (1972), Labov y Waletzky (1967) y Peterson y McCabe (1983, cit. por López Morales, 2004), Reyes (1996) estableció la existencia de siete patrones narrativos, cuya taxonomía utilizaremos en el análisis de las diferencias de género en las narraciones de hablantes juveniles de Santiago de Chile. Concretamente, la propuesta de Reyes (1996) puede resumirse del modo siguiente:

1) Patrón clásico: la narración se va construyendo hasta un punto máximo, se refuerza evaluativamente en él, y entonces se resuelve.

2) Patrón que termina en el punto máximo: la narración se va construyendo hasta un punto máximo y entonces termina. No hay resolución.

3) Patrón de saltos múltiples: la narración salta de un evento a otros dentro de una experiencia integrada, dejando fuera eventos importantes que deben ser inferidos por el oyente.

4) Patrón cronológico: la narración es una simple descripción sucesiva de eventos.

5) Patrón empobrecido: la narración está constituida por muy pocas oraciones para poder reconocer un punto máximo, o bien, la narración reitera y evalúa extensamente solo dos eventos.

6) Patrón desorientado: la narración es demasiado confusa o desorientada como para que el oyente pueda comprenderla.

7) Patrón misceláneo: cualquier narración que no entre en alguna de las categorías anteriores (Adaptado de Reyes 1996:112).

\subsection{LAS VARIABLES SOCIOLINGÜÍSTICAS Y LOS FACTORES SOCIALES}

El interés de la sociolingüística por el estudio de las variables lingüísticas es fundamental, ya que el desarrollo de esta interdisciplina se basa en que gran parte de los hechos de variación lingüística no varía de manera azarosa, sino que en forma sistemática, si se los correlaciona con variables demosociales como la clase social, la edad y el sexo-género. Esto implica que el hecho de que las variables lingüísticas estén en covariación sistemática con las variables permite a los individuos situarse en un determinado espacio del espectro social. A este respecto, Coates señala que:

El uso de las variables lingüísticas es una de las formas en que los hablantes pueden ubicarse en el espacio social. Es decir, aunque desde el punto de vista lingüístico las variables lingüísticas sean formas 
equivalentes para referirse a algo en particular, son diferentes desde una perspectiva social (Coates 2009: 89).

Según Moreno Fernández (1998), la investigación sociolingüística ha permitido determinar que las variables sociales que influyen sobre la variación lingüística lo hacen de una forma específica en cada comunidad de habla y respecto de fenómenos lingüísticos concretos. Por su parte, Blas Arroyo (2005) señala que no es posible reconocer de antemano qué tipo de variables sociales van a actuar sobre la variación lingüística, debido a que la variabilidad no tiene por qué manifestarse de la misma forma en comunidades de habla diferentes y, además, porque estos mismos factores no se configuran de la misma forma en sociedades distintas. Así ocurre con las variables sexo, edad y clase social. Como ya lo señalamos, el factor sexo, en primer lugar, y la clase social, en segundo término, serán las variables independientes empleadas en esta investigación.

\subsubsection{Algunas precisiones sobre la variable sexo-género}

En relación con el factor sexo, consideramos necesario precisar que dicha variable se ha considerado, tradicionalmente, según López Morales (2004), por un lado, en lo referido al sexo como categoría biológica y, por otro, en lo que respecta al género en tanto construcción sociocultural. En este sentido, Cheshire (2003) señala que el término "sexo" ha sido empleado para referirse a la distinción fisiológica entre mujeres y hombres, mientras que "género" lo ha sido para la elaboración social y cultural de sexos diferentes, en un proceso vinculado con los roles sociales, las oportunidades y las expectativas. Sin embargo, de acuerdo con esta autora, puesto que el proceso comienza con el nacimiento, podría discutirse si "género" es el término más apropiado para esta categoría en lugar de "sexo". No obstante, ambos términos se encuentran en la literatura del variacionismo usados a veces en una manera, al parecer, indistinta pero, en otras ocasiones, empleados para distinguir entre las características biológicas y los factores sociales de los sujetos.

\subsection{LOS ESTUDIOS SOBRE SEXO-GÉNERO Y DISCURSO}

Según Moreno Fernández (1998), la mayor parte de lo que hoy sabemos acerca de la conducta lingüística de hombres y mujeres se lo debemos a la sociolingüística. Los estudios sobre la relación entre género y discurso surgen en la década de los setenta, específicamente, en 1975. Prieto y San Martín (2002-2003) señalan que estos primeros trabajos eran un intento por 
caracterizar el habla de mujeres y hombres, pero sin trascender al ámbito de la explicación de estas características. En etapas siguientes, en cambio, los enfoques son más bien explicativos, donde destacan investigaciones como las de Tannen (1990a), quien enfatiza el carácter intercultural de la interacción conversacional al plantear la tesis de que los hombres y las mujeres utilizan estilos conversacionales diferentes. En este sentido, el interés del hombre radicaría en comunicar información y mantener la independencia y el estatus frente a su interlocutor, mientras que las mujeres estarían más preocupadas de establecer y mantener una relación a nivel interpersonal. En atención a este principio, es importante precisar que en esta investigación será central el enfoque de las diferencias culturales, puesto que la idea subyacente a las diferencias de género en el discurso narrativo juvenil es que, tal como lo precisan estudios como los de Tannen (1990b), los hombres y las mujeres poseen estilos conversacionales distintos no por azar, sino porque sus procesos de socialización son, asimismo, diferentes.

La incidencia de la variable de sexo-género en el discurso es un problema que ha sido abordado de un modo especial en la lingüística norteamericana, entre otros, por Lakoff (1973), Coates (2009 [1987]) y Tannen (1990a). Asimismo, de gran utilidad para los propósitos de esta investigación resultó ser el panorama del desarrollo histórico de los estudios sobre género y discurso que presentan Prieto y San Martín (2002-2003). Según estos autores, los factores que explicarían los rasgos atribuidos al habla femenina en la investigación empírica podrían explicarse a través de la propuesta de Coates basada en una apreciación del trabajo de Jones (1980) sobre el cotilleo femenino. Estos factores serían: 1) la situación comunicativa, 2) los participantes, 3) el tópico y 4) las funciones o metas del acto comunicativo. Otro aspecto importante en relación con los estudios sobre el discurso narrativo y las diferencias de género es el que se vincula con el discurso referido y la oralidad. En su investigación sobre las diferencias de género en el empleo del discurso referido, los autores antes citados concluyen que existe un uso preponderante de estilo directo en las interacciones femeninas, lo que podría explicarse por una reminiscencia de la "oralidad primaria" en una sociedad que ha devenido en una cultura preponderantemente escrita ${ }^{1}$;

Según estos autores, la oralidad primaria no se condice con un retraso en la marcha hacia la racionalidad y la modernidad, porque en el ámbito sociológico, el paso a la modernidad ha sido descrito empleando la conceptualización del sociólogo alemán Ferdinand Tönnies, como un tránsito de la Gemeinschaft-que define el tipo de relaciones sociales entre individuos con vínculos interpersonales estrechos e íntimos, preocupación por el bienestar de los demás y cooperación y confianza mutuas, además del valor asignado a los derechos y obligaciones tradicionales- a la Gesellschaft-que define un tipo de sociedad en la que las relaciones 
es decir, entre los representantes del género masculino se habría perdido este rasgo de oralidad como consecuencia del paso del orden tradicional a la modernidad, mientras que entre las representantes del género femenino dicho rasgo pervive con mayor frecuencia al haber accedido las mujeres a la educación formal y a la comunicación escrita con bastante posterioridad a los varones.

A partir de los aportes teóricos de los autores antes señalados, esta investigación se centrará en el análisis de las diferencias de género en el empleo de los patrones narrativos en un subtipo específico del discurso narrativo juvenil en Santiago de Chile: las narraciones de experiencias personales.

\section{METODOLOGÍA}

\subsection{CORPus}

La muestra que sirve de base para el presente estudio corresponde a 64 entrevistas sociolingüísticas realizadas en la cátedra de Sociolingüística, a cargo del profesor Dr. Luis Prieto Vera, de las Licenciaturas en Lengua y Literatura Hispánicas y Lengua y Literatura Inglesas de la Universidad de Chile, de cada una de las cuales se extrajo el segmento narrativo mejor desarrollado por los entrevistados. Las entrevistas fueron realizadas a hombres y mujeres jóvenes con características socio-demográficas congruentes y, aplicando los supuestos metodológicos sugeridos por Labov (1992); en dichas situaciones los entrevistadores debían tratar de superar la "paradoja del observador" ${ }^{2}$ consiguiendo, de esta forma, una muestra

sociales se caracterizan por la competencia, el interés propio, la eficiencia, el progreso y la especialización, además del desapego de los lazos tradicionales.

2 Según Labov (1992), el objetivo de la investigación lingüística de una comunidad de habla consiste en estudiar la forma cómo habla la gente cuando no está siendo sistemáticamente observada; sin embargo, solo podemos obtener tales datos mediante la observación sistemática. A esto es a lo que se le ha denominado paradoja del observador. Este dilema se soluciona encontrando la manera de completar la entrevista formal con otros datos, o cambiar la estructura de la situación de la entrevista de una u otra manera. En este sentido, una forma de superar la paradoja consiste en romper las constricciones de la situación de entrevista mediante diversos procedimientos que puedan distraer la atención del sujeto en su propio discurso y puedan hacer brotar su habla más vernácula. Esto puede llevarse a cabo mediante intervalos y cortes definidos de tal manera que el entrevistado suponga inconscientemente que en ese momento 
significativa de discurso natural grabado (estilo vernacular) de hablantes representativos de la comunidad de habla en estudio. El cuestionario utilizado para la realización de las mencionadas entrevistas consta de una sección cuya función es elicitar las narraciones de experiencias personales que conforman el corpus de nuestra investigación. En concreto, se trata de 64 entrevistas grabadas, realizadas a jóvenes santiaguinos de entre 20 y 34 años, estratificados por sexo y nivel socioeconómico. Las entrevistas que conforman la muestra del estudio fueron grabadas entre 2005 y 2009 y conforman una parte del corpus del proyecto de Estudio Sociolingüístico del Español de Chile (ESECH) ${ }^{3}$.

\subsubsection{Procedimiento de estratificación social utilizado}

Para la estratificación de los sujetos que conformaron la muestra del estudio se empleó el sistema de adscripción de estatus social empleada en ESECH que, a su vez, complementó la propuesta de Prieto (1995-1996) con la Descripción Básica de los Niveles Sociales del estudio de mercado realizado en 2007 por ICCOM (Instituto Consultor en Comercialización y Mercado Limitada). Siguiendo a Prieto (1995-1996), la muestra de estudio se estratificó convenientemente, utilizando una escala de adscripción de estatus socioeconómico, basada en la combinación de cuatro variables: 1) nivel educacional, 2) profesión u ocupación, 3) comuna de residencia, y 4) ingreso familiar mensual. Dicha escala incluye cuatro estratos socioeconómicos: bajo, medio bajo, medio y medio alto, para cuya diferencia se cauteló la consistencia entre las variables antes mencionadas de manera que respondieran a lo que Lenski (1954) denominara congruencia de status ${ }^{4}$.

no está siendo observado. Asimismo, siguiendo a Labov, también pueden implicarse temas en que los informantes puedan reproducir emociones intensas que han experimentado en el pasado, por ejemplo, planteando preguntas relativas al "peligro de muerte". Los relatos aportados como respuesta a esta pregunta, según este autor, presentan casi siempre un cambio de estilo respecto al discurso más cuidado aproximándose al más vernáculo.

3 El equipo PRESEEA (Proyecto para el Estudio Sociolingüístico del Español de España y América) Santiago de Chile está compuesto por los profesores de la Universidad de Chile, Alfredo Matus y Luis Prieto (coordinadores), Abelardo San Martín y Silvana Guerrero (asistentes). El proyecto ESECH corresponde a un grupo de estudio de la Universidad de Chile. Cabe mencionar que 36 de las entrevistas que conforman nuestro corpus forman parte, a su vez, del corpus PRESEEA Santiago de Chile; sin embargo, para los propósitos de este estudio dichas entrevistas se post-estratificaron según la metodología de ESECH.

4 Lenski (1954) acuñó el término cristalización o congruencia de status. Se considera que un individuo es congruente con su estatus cuando las puntuaciones obtenidas en las diferentes dimensiones usadas para medir el estatus son más o menos iguales, independiente 


\subsubsection{Población y muestra}

En la presente investigación se consideró la población constituida por hombres y mujeres de la Región Metropolitana, de entre 20 y 34 años de edad, que corresponden al segmento etario más joven de la población santiaguina. $\mathrm{Al}$ respecto, los criterios de asignación de hablante nativo de Santiago de Chile son los siguientes: 1) haber nacido y residido en forma ininterrumpida en Santiago; 2) haber residido en forma ininterrumpida en Santiago desde los cinco años de edad y 3 ) haber nacido en Santiago y haber residido en Santiago la mayor parte de sus vidas, salvo por periodos que sumados no superen los cuatro años en el tramo de 50 años y más y los tres años en el tramo de 35 a 49 años.

El cuestionario se aplicó a una muestra del tipo denominado "muestra por cuotas", en la que se divide a la población en estratos o categorías y se asigna una cuota a cada uno de los distintos estratos. La muestra así conformada comprende un total de 64 individuos, distribuidos proporcionalmente como se indica en la Tabla 1 que se incluye a continuación.

TABLA 1: Distribución de sujetos de la muestra por nivel socioeconómico, sexo y edad según ESECH

\begin{tabular}{|c|c|c|c|}
\hline \multicolumn{4}{|c|}{ Grupo de edad } \\
\hline Nivel socioeconómico & \multicolumn{2}{|c|}{$20-34$} & Totales \\
\cline { 2 - 4 } & $\mathrm{H}$ & $\mathrm{M}$ & \\
\hline Medio alto & 8 & 8 & 16 \\
\hline Medio & 8 & 8 & 16 \\
\hline Medio bajo & 8 & 8 & 16 \\
\hline Bajo & 8 & 8 & 16 \\
\hline Totales & 32 & 32 & 64 \\
\hline
\end{tabular}

En la Tabla 2, por su parte, se presentan las características socio-demográficas de cada uno de los integrantes de la muestra, clasificados de acuerdo con su estrato socioeconómico y su sexo-género.

de que sus rangos sean altos, bajos o estén en una extensión media. Cuando las puntuaciones del individuo son muy diferentes, se habla de incongruencia de status 
TABLA 2: Características socio-demográficas de los integrantes de la muestra

\begin{tabular}{|c|c|c|c|c|c|c|c|}
\hline $\begin{array}{c}\text { Grupo } \\
\text { de edad }\end{array}$ & Estrato & Sexo & Código & Edad & $\begin{array}{l}\text { Nivel } \\
\text { educa- } \\
\text { cional }\end{array}$ & Profesión u ocupación & $\begin{array}{l}\text { Comuna de } \\
\text { residencia }\end{array}$ \\
\hline \multirow{48}{*}{ 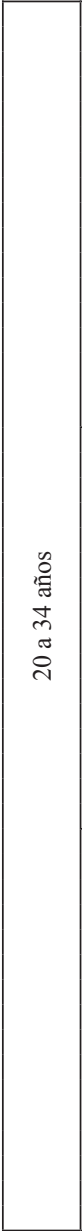 } & \multirow{16}{*}{  } & \multirow{8}{*}{ 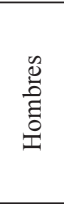 } & $\mathrm{H} 181^{5}$ & 23 & $\mathrm{UI}^{6}$ & Estudiante de Diseño Industrial & Providencia \\
\hline & & & H182 & 24 & UC & Licenciado en Derecho & Peñalolén \\
\hline & & & H183 & 26 & UI & Operador de Sistemas Informáticos & Peñalolén \\
\hline & & & $\mathrm{H} 184$ & 25 & UI & Estudiante de Literatura & Vitacura \\
\hline & & & $\mathrm{H} 185$ & 22 & UI & Estudiante de Filosofía & Nuñoa \\
\hline & & & $\mathrm{H} 186$ & 25 & UI & Estudiante de Ingeniería Industrial & Peñalolén \\
\hline & & & H187 & 26 & $\mathrm{UC}$ & Constructor Civil & Providencia \\
\hline & & & H188 & 21 & UI & Estudiante de Historia & Nuñoa \\
\hline & & \multirow{8}{*}{ 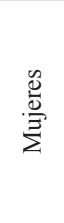 } & M191 & 31 & $\mathrm{UC}$ & Psicóloga & Nuñoa \\
\hline & & & M192 & 21 & UI & Estudiante de Medicina & Las Condes \\
\hline & & & M193 & 25 & $\mathrm{UC}$ & Licenciada en Inglés & Nuñoa \\
\hline & & & M194 & 21 & UI & Estudiante de Terapia Ocupacional & Nuñoa \\
\hline & & & M195 & 28 & $\mathrm{UC}$ & Médico psiquiatra & Providencia \\
\hline & & & M196 & 21 & UI & Estudiante de Teatro & Nuñoa \\
\hline & & & M197 & 23 & UI & Estudiante de Filosofía & Las Condes \\
\hline & & & M198 & 26 & UI & $\begin{array}{l}\text { Estudiante de Licenciatura } \\
\text { en Criminalística }\end{array}$ & Peñalolén \\
\hline & \multirow{16}{*}{ 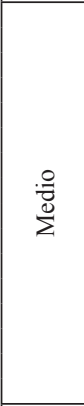 } & \multirow{8}{*}{ 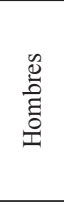 } & $\mathrm{H} 121$ & 23 & $\mathrm{UI}$ & Estudiante de Física & La Florida \\
\hline & & & $\mathrm{H} 122$ & 26 & $\mathrm{UC}$ & Profesor de Educación Física & Maipú \\
\hline & & & H123 & 22 & UI & Estudiante de Pedagogía en Castellano & Nuñoa \\
\hline & & & $\mathrm{H} 124$ & 25 & UI & Estudiante de Inglés & La Florida \\
\hline & & & $\mathrm{H} 125$ & 22 & TPI & Estudiante de Geografía & Santiago \\
\hline & & & H126 & 25 & UI & Estudiante de Arquitectura & Santiago \\
\hline & & & $\mathrm{H} 127$ & 22 & UI & Estudiante de Diseño Gráfico & Macul \\
\hline & & & H128 & 21 & UI & Estudiante de Arquitectura & Maipú \\
\hline & & \multirow{8}{*}{$\frac{\mathscr{d}}{\stackrel{0}{0}}$} & M131 & 24 & TPC & Técnico paramédico & Santiago \\
\hline & & & M132 & 30 & TPC & Administradora de Local Comercial & Las Condes \\
\hline & & & M133 & 25 & $\mathrm{UC}$ & Profesora de Inglés & Maipú \\
\hline & & & M134 & 25 & $\mathrm{UC}$ & Parvularia & Las Condes \\
\hline & & & M135 & 28 & UI & Estudiante de Inglés & Santiago \\
\hline & & & M136 & 21 & UI & Estudiante de Fonoaudiología & Santiago \\
\hline & & & M137 & 26 & UI & Estudiante de Enfermería & Nuñoa \\
\hline & & & M138 & 23 & UI & Estudiante de Medicina Veterinaria & Puente Alto \\
\hline & \multirow{16}{*}{  } & \multirow{8}{*}{  } & $\mathrm{H} 061$ & 29 & $\mathrm{MC}$ & Chofer de colectivo & Puente Alto \\
\hline & & & $\mathrm{H} 062$ & 32 & $\mathrm{MC}$ & Operario de bodega & Maipú \\
\hline & & & H063 & 20 & $\mathrm{MC}$ & Reponedor de supermercado & La Florida \\
\hline & & & $\mathrm{H} 064$ & 32 & $\mathrm{MC}$ & Transportista & San Bernardo \\
\hline & & & $\mathrm{H} 065$ & 25 & TPC & Fotocopiador & Estación Central \\
\hline & & & $\mathrm{H} 066$ & 30 & TPI & Estudiante de Informática & Puente Alto \\
\hline & & & $\mathrm{H} 067$ & 25 & $\overline{M C}$ & Vendedor & Puente Alto \\
\hline & & & H068 & 20 & UIC & Cesante & La Florida \\
\hline & & \multirow{8}{*}{ 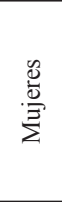 } & M071 & 32 & $\mathrm{MC}$ & Lavandera & La Cisterna \\
\hline & & & M072 & 28 & TPC & Esteticista & La Florida \\
\hline & & & M973 & 24 & MTPC & Ayudante de cocina & San Ramón \\
\hline & & & M074 & 20 & TPC & Cesante & Quinta Normal \\
\hline & & & M075 & 32 & $\mathrm{MC}$ & Vendedora & Puente Alto \\
\hline & & & M076 & 28 & $\mathrm{MC}$ & Contadora & Quinta Normal \\
\hline & & & M077 & 20 & $\mathrm{MC}$ & Promotora & Puente Alto \\
\hline & & & M078 & 22 & TPI & Estudiante Técnico en Enfermería & Puente Alto \\
\hline
\end{tabular}

5 El código de los sujetos tiene las siguientes equivalencias: sexo: $\mathrm{H}$ (hombre) y $\mathrm{M}$ (mujer). El número que le sigue a dichas equivalencias corresponde a la numeración correlativa que se le asignó a los informantes en el corpus del ESECH.

6 Las siglas de esta columna tienen las siguientes equivalencias: $\mathrm{UC}=$ educación universitaria completa; $\mathrm{UI}=$ educación universitaria incompleta; $\mathrm{UIC}=$ educación universitaria incompleta congelada; TPC $=$ educación técnica-profesional completa; TPI = educación técnicaprofesional incompleta; $\mathrm{MC}$ = educación media completa; $\mathrm{MI}=$ educación media incompleta; MTPC = educación media-técnica profesional completa; MTPI = educación media-técnica profesional incompleta; $\mathrm{BC}=$ educación básica completa; $\mathrm{BI}=$ educación básica incompleta. 


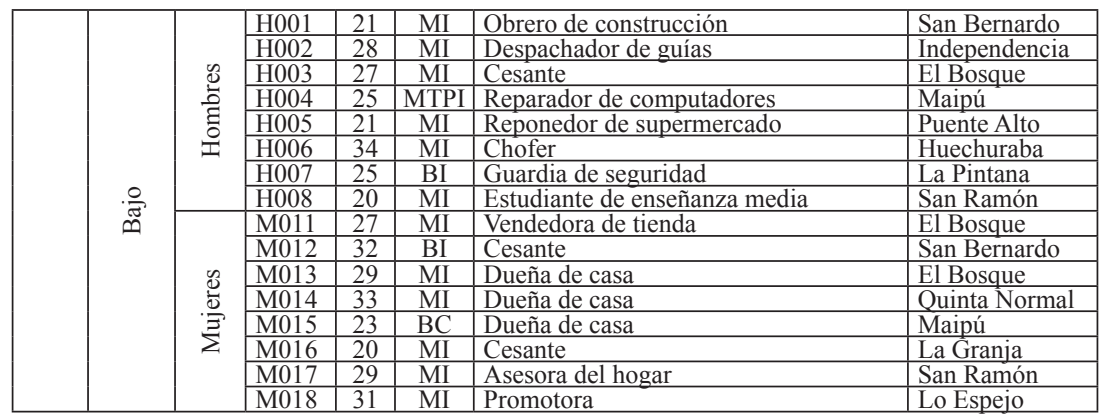

\section{PRESENTACIÓN Y ANÁLISIS DE LOS RESULTADOS}

Para facilitar la comprensión de los fenómenos analizados en esta sección se utilizarán las siguientes convenciones en la presentación de los ejemplos:

a. Se transcribirá cada ejemplo en forma de cita incluyendo la cantidad de contexto necesaria para su comprensión ${ }^{7}$.

b. Cada tipo de patrón narrativo será debidamente identificado y comentado.

c. Al final de cada cita se indicará, entre paréntesis, el código del sujeto que la emitió.

\subsection{AnÁlisis DE PATRONES NARRATIVOS}

Reyes (1996) señala que en la estructura de las narraciones, podemos determinar la existencia de siete patrones narrativos (ver 2.1.1). En este sentido, nuestro estudio está enfocado en detectar la existencia de diferencias

7 Respecto de la transcripción de los ejemplos, es necesario señalar las siguientes convenciones gráficas: 1) $\mathrm{E}=$ entrevistador e I = informante; 2) el texto correspondiente a cada entrevista se transcribe en ortografía convencional, incluidos los acentos gráficos. Las palabras que presentan elisiones en su pronunciación se completan en su escritura con paréntesis cuadrados. En el caso de las aspiraciones, estas se marcarán con $h ; 3$ ) para la mejor comprensión de los ejemplos que hemos incluido en este artículo, se ha suprimido el sistema de etiquetas pertenecientes al Standard Generalized Markup Language (SGML) contenidas en el Text Encoding Initiative (TEI), que se emplean en la transcripción de las entrevistas pertenecientes al PRESEEA, excepto las pausas. 
de género en relación con los patrones narrativos observados en los sujetos que conforman la muestra. Por consiguiente, en lo que sigue, presentamos la Tabla 3 y el Gráfico 1 donde se muestra la frecuencia general de los patrones narrativos registrados en el corpus.

TABLA 3: Tabla de frecuencia de patrones narrativos registrados en el corpus

\begin{tabular}{|l|c|c|c|c|c|c|c|}
\hline \multirow{2}{*}{ Sexo } & \multicolumn{7}{|c|}{ Total patrones narrativos } \\
\cline { 2 - 8 } & $\begin{array}{c}\text { Patrón } \\
\text { clásico }\end{array}$ & $\begin{array}{c}\text { Patrón que } \\
\text { termina en } \\
\text { el punto } \\
\text { máximo }\end{array}$ & $\begin{array}{c}\text { Patrón } \\
\text { de saltos } \\
\text { múltiples }\end{array}$ & $\begin{array}{c}\text { Patrón } \\
\text { cronológico }\end{array}$ & $\begin{array}{c}\text { Patrón } \\
\text { empobrecido }\end{array}$ & $\begin{array}{c}\text { Patrón } \\
\text { desorientado }\end{array}$ & $\begin{array}{c}\text { Patrón } \\
\text { misceláneo }\end{array}$ \\
\hline Hombres & 20 & 2 & 6 & 0 & 4 & 0 & 0 \\
\hline Mujeres & 22 & 2 & 4 & 0 & 3 & 1 & 0 \\
\hline Totales & 42 & 4 & 10 & 0 & 7 & 1 & 0 \\
\hline
\end{tabular}

Gráfico 1: Gráfico de frecuencia de patrones narrativos registrados en el corpus

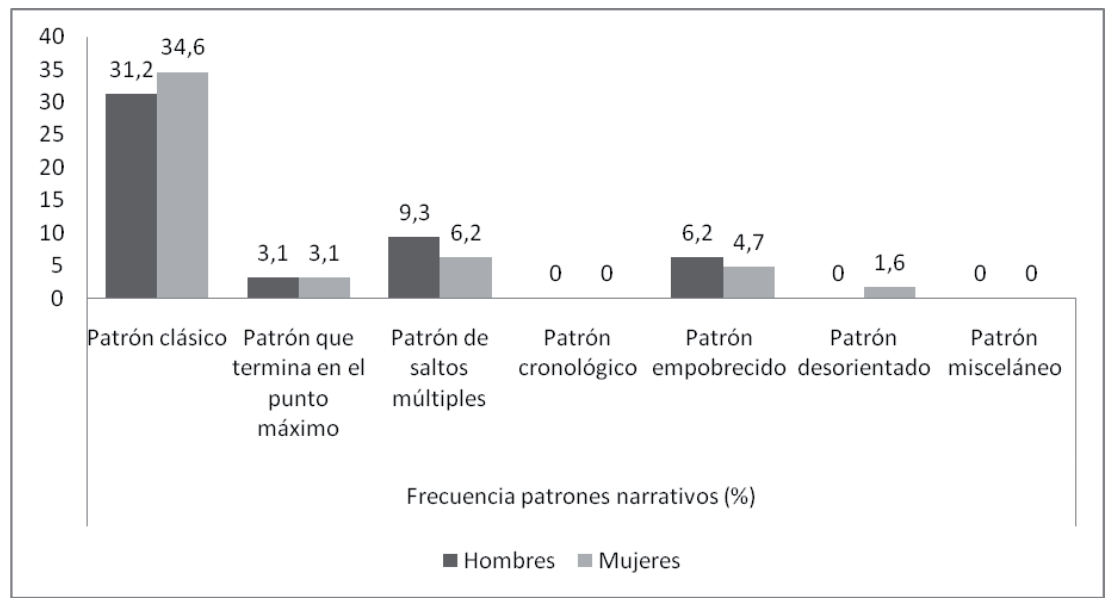

De acuerdo con los datos proporcionados en la Tabla 3 y en el Gráfico 1 , en relación con las diferencias de género en la frecuencia de patrones narrativos registrada en los relatos recopilados, pudimos constatar algunas leves diferencias en cuanto a la cantidad de patrones narrativos registrados entre los hablantes masculinos y femeninos en estudio. En la mayoría de las ocasiones, nuestros hablantes cuentan sus historias mediante el patrón clásico, es decir, la narración se va construyendo hasta un punto máximo, 
se refuerza evaluativamente en él, y entonces se resuelve; de hecho, el $65,8 \%(42 / 64)$ narraciones se construyen sobre la base de este patrón. De ellas, el 34,6\% (22/42) son empleados por mujeres y el 31,2\% (20/42) por hombres. Le sigue en frecuencia el patrón de saltos múltiples presente en el 15,5\% (10/64) de los casos, de los cuales el 9,3\% (6/10) corresponde a mujeres y 6,2 (4/10) a hombres. El patrón empobrecido se ubica en el tercer lugar con el 10,9\% (7/64) de los patrones narrativos registrados en el corpus, en cuyo caso el 6,2\% (4/7) es empleado por hombres y el $4,7 \%$ (3/7) por mujeres. Posteriormente, se encuentra el patrón que termina en el punto máximo con el 6,2\% (4/64) de las apariciones en el total de relatos de la muestra con igual frecuencia para hombres y mujeres: $3,1 \%(2 / 4)$ en cada caso y, finalmente, se ubica el patrón desorientado con apenas el 1,6\% $(1 / 64)$ de frecuencia de aparición en las narraciones que sirvieron de base a esta investigación; dicho patrón narrativo fue empleado solo por una mujer. No se observó la presencia ni del patrón cronológico ni del misceláneo. Sin embargo, es preciso destacar que aunque la mayoría de nuestros relatos se construye de manera cronológica, no han sido catalogados dentro de este patrón debido a que no son una simple descripción sucesiva de eventos, sino que responden a patrones narrativos de mayor complejidad.

No menos interesantes que las diferencias mencionadas anteriormente son aquellas relacionadas con la variable estrato social de los informantes junto con su sexo-género. Por ello, en lo que sigue, presentamos, en la Tabla 4 y el Gráfico 2, la frecuencia de patrones narrativos incluyendo ambas variables.

TABLA 4: Frecuencia de patrones narrativos registrados en el corpus en correlación con la variable estrato social de los sujetos

\begin{tabular}{|c|c|c|c|c|c|c|c|c|}
\hline \multirow[b]{2}{*}{ Estrato } & \multirow[b]{2}{*}{ Sexo } & \multicolumn{7}{|c|}{ Total patrones narrativos } \\
\hline & & $\begin{array}{l}\text { Patrón } \\
\text { clásico }\end{array}$ & $\begin{array}{l}\text { Patrón que } \\
\text { termina en } \\
\text { el punto } \\
\text { máximo }\end{array}$ & $\begin{array}{l}\text { Patrón } \\
\text { de saltos } \\
\text { múltiples }\end{array}$ & $\begin{array}{l}\text { Patrón } \\
\text { crono- } \\
\text { lógico }\end{array}$ & $\begin{array}{l}\text { Patrón em- } \\
\text { pobrecido }\end{array}$ & $\begin{array}{l}\text { Patrón des- } \\
\text { orientado }\end{array}$ & $\begin{array}{l}\text { Patrón } \\
\text { misceláneo }\end{array}$ \\
\hline \multirow{2}{*}{$\begin{array}{l}\text { Medio } \\
\text { alto }\end{array}$} & Hombres & 5 & 0 & 2 & 0 & 1 & 0 & 0 \\
\hline & Mujeres & 4 & 1 & 1 & 0 & 1 & 1 & 0 \\
\hline \multirow[t]{2}{*}{ Medio } & Hombres & 4 & 1 & 2 & 0 & 1 & 0 & 0 \\
\hline & Mujeres & 4 & 1 & 2 & 0 & 1 & 0 & 0 \\
\hline \multirow{2}{*}{$\begin{array}{l}\text { Medio } \\
\text { bajo }\end{array}$} & Hombres & 4 & 1 & 2 & 0 & 1 & 0 & 0 \\
\hline & Mujeres & 7 & 0 & 1 & 0 & 0 & 0 & 0 \\
\hline \multirow[t]{2}{*}{ Bajo } & Hombres & 7 & 0 & 0 & 0 & 1 & 0 & 0 \\
\hline & Mujeres & 7 & 0 & 0 & 0 & 1 & 0 & 0 \\
\hline
\end{tabular}


Gráfico 2: Gráfico general de frecuencia de patrones narrativos registrados en el corpus en correlación con la variable estrato social de los sujetos

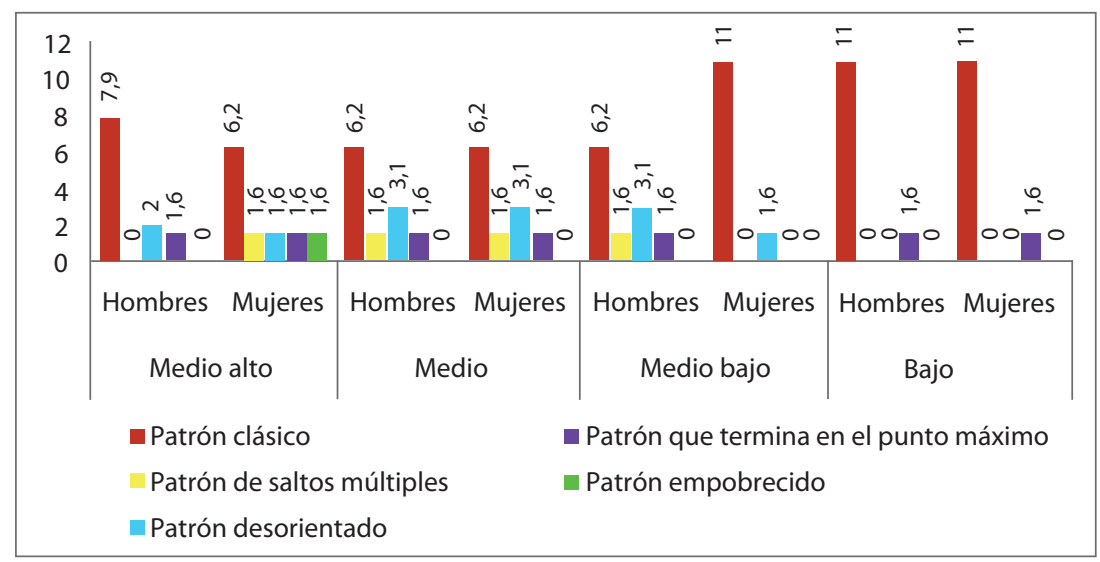

Como podemos observar en la Tabla 4 y en el Gráfico 2, la frecuencia de patrones narrativos, considerando el estrato social de los informantes, da cuenta de que no existen grandes diferencias de género dentro de cada estrato. De hecho, las frecuencias de empleo de los distintos patrones narrativos analizados, comparando hombres con mujeres en los cuatro estratos socioeconómicos examinados, son prácticamente idénticas. No obstante lo anterior, a partir de los datos presentados, debemos mencionar que las mayores diferencias en cuanto a la frecuencia de empleo de los patrones narrativos se detectaron, más que a nivel de la variable sexo, en el nivel de estrato social de los sujetos que conforman nuestra muestra; esto es, en los estratos bajo y medio bajo se presentan los índices más elevados de empleo del patrón clásico ( $22 \%$ en el estrato bajo y $17,1 \%$ para el estrato medio bajo), en desmedro de los estratos medio alto y medio, que representan el $14 \%$ y $12,4 \%$, respectivamente. Estos antecedentes permiten afirmar que son los hablantes de los extremos inferiores de la escala socioeconómica quienes, al narrar, lo hacen con mayor fidelidad a los hechos que realmente ocurrieron, construyendo sus narraciones hasta llegar al punto máximo para, finalmente, precisar la resolución de los hechos. Asimismo, esta afirmación se comprueba al observar que la presencia, aunque mínima, del patrón que termina en el punto máximo, del de saltos múltiples, del empobrecido y del desorientado es mayor en los extremos superiores de la escala de estratificación social, en comparación con el estrato bajo que, en general, no registra casos de relatos construidos en base a dichos patrones narrativos. 
Con el fin de ilustrar los antecedentes anteriores, a continuación, presentamos los ejemplos más representativos de los patrones narrativos que registraron un mayor índice de frecuencia en los relatos en estudio.

(1) I: eeeh a bueno ¿en el colegio por ejemplo decíh tú? eh mmm sí poh/igual nosotroh éramoh súper desordenadoh mi curso éramoh super unidoh y desordenadoh me acuerdo que teniamoh un botiquín/ y eeh obviamente uno en loh colegioh loh botiquineh no se pone no se echa na[da] lo botiquineh to[d]o vacío y lo ocupábamoh pa[ra] dihtinta cosa/ por ejemplo/ al principio era la cabina telefónica/ un compañero trajo el teléfono del hermano chico y lo puso ahí en el en el botiquín poh/ entonceh eh por ejemplo ehtábamos en claseh y to[d]o empezábamoh ¡teléfono! ¡teléfono! y la profesora tenía que pararse y contehtar el teléfono y nosostroh la, otro compañero le contehtaba cuetioneh/ despuéh era fax/ porque fuimoh progresando / y también mih compañeroh leh ponían papeleh con siempre con la mihma profesora la profe de hihtoria y como que noh aguantaba to[d]a la cuehtioneh que hacíamoh y le ponían le ponían aparte que era bonita ella entonceh le ponían en el fax mih compañero le ponían por ejemplo profe hoy día se ve linda con el pañuelo rojo/ mi entonceh empezábamoh fax fax fax fax /y la profe se tenía que parar a contehtar a ver qué papel había poh y lo leía y le pedíamoh que lo leyera en voh alta/ y se ponía roja de vergüenza y un día un día de invierno hacía mucho frío en el colegio en la sala/ y una compañera le dice a otro compañero/ oye sabíh que tengo frío / eh ya no te preocupí yo te voy a ayudar y mi compañero no encontró nada mejor de que agarrar papel lo mete dentro del botiquín y le prende fuego a la cuestión empezó a salir humo llegó el inhpector indignadoy ahí noh y ahí noh sacaron el botiquín poh/ lo tuvieron que sacar porque ya era mucho el ehcándalo con el famoso botiquín (M075, estrato medio bajo).

En el ejemplo anterior podemos observar cómo se va construyendo la historia de acuerdo con el patrón clásico. En este caso, se trata del relato de una mujer de estrato medio bajo, cuya historia se desarrolla progresivamente hasta el punto máximo. Primero, la entrevistada orienta los hechos; su anécdota tuvo lugar en el colegio y, en concreto, tiene relación con el botiquín del curso; de hecho, la historia comienza cuando ella ejemplifica con casos concretos el uso que le daban al botiquín, evaluándose constantemente ("éramos súper desordenados", "éramos súper unidos y desordenados", "porque fuimos progresando", "aparte que era bonita", "y era mucho el escándalo", etc.). Luego, mediante los ejemplos de las distintas situaciones que se generaban con el botiquín, llega al punto máximo del relato (una de sus compañeras tiene frío) y, finalmente, los hechos se resuelven (queman un papel dentro del botiquín y debido al caos que se generó, se lo quitan). 
(2) E: y / ¿recuerdah alguna anécdota graciosa de / del colegio / o de teatro?

I: de teatro sí poh muchah

E: cuéntame alguna

I: esa la del cuando ehtábamoh actuando / ehtábamoh presentando la obra y falta el el el falta el...

E: el gato

I: el gato // y el Nico hizo waw / eso / eso me acuerdo/ siempre me acuerdo de eso (M016, estrato bajo).

En el ejemplo (2), por su parte, si bien la historia respeta el orden cronológico de los sucesos (se va a contar una anécdota acontecida mientras se realiza una obra de teatro y falta uno de los personajes de la obra), no se puede verificar la existencia de la resolución, pues la entrevistada no relata qué ocurrió una vez que su compañero reemplaza la voz del gato, por lo tanto, constituye una narración que finaliza en el punto máximo.

(3) I: cuál diría yo que ha sido el suhto más grande de mi vida / difícil / a ver / bueno un suhto grande/ yo diría que el máh grande / que he tenido / ehm eh/ bueno / fue cuando estaba de gira de estudioh en Brasil / que / tuvimoh un problema con / con / tuve un problema ya poh / con / que me llevaron detenido digamoh/y era bahtante / en realidad ehtaba bahtante asuhtado porque bueno no andaba con documentación / y ahí /y / y no sabía bien que me iba a pasar / digamoh / aparte de toda la presión que uno siente por estar con tu curso / y que / qué van a decir / y todo el cuento / ademáh de eso / no saber que / que te van a hacer / me dijeron que me iban a mandar a Chile de vuelta / así como muy de cortá / no me creían / decían que había ido a robar / era muy penca

E: pero por qué / por qué / te arrehtaron?

I: me detuvieron /por bueno por entrar a una casa sin permiso

E: pero y cómo / y cómo salihte al final de eso?

I: me encontraron los / digamoh el grupo / con la guía turística y el rehto / del grupo / me/ notificaron/bueno allá la policía es la policía militar / no es la / no es / como acá digamoh / eh / dieron el aviso / y se contactaron / y cacharon donde estaba / $\mathrm{mm}$ si yo diría que me acuerdo de ese susto no mah

E: pero / pero después no pasó a mayores

I: no / por suerte no / entiendo que ni siquiera tengo antecedenteh penaleh (H121, estrato medio). 
Por otro lado, en (3), el narrador utiliza el patrón de saltos múltiples para construir su relato, pues, si bien comienza haciendo un resumen de los hechos (estaban en Brasil en su viaje de estudios y se lo llevaron detenido), relata, en lo sucesivo, prácticamente el final de su aventura (le dijeron que lo mandarían de regreso a Chile), mediado de varias evaluaciones ("fue un suhto grande", "el suhto máh grande", "ehtaba bahtante asuhtado"). Solo con la ayuda del entrevistador, el informante, logra construir la historia tal como ocurrió, pues luego de haber narrado la resolución, cuenta que fue lo que le sucedió y cómo se resolvió (entró a una casa sin autorización de sus dueños. Posteriormente, con ayuda de la guía turística, lo encontraron y quedó en libertad), aunque de manera muy general, saltando de un episodio a otro.

(4) E.: eehm/ al ingresar al primer año de tu carrera/ ¿te hicieron alguna activida[d] de recepción?/ no sé poh/ un mechoneo o algo así

I.: sí/ si hubo mechoneo/ pero no fue muy// muy violento/ fue na[d]a/ noh hicieron noh quitaron la mochila/ la guardaron en una sala// eeh/ y dehpu[és] y dehpuéh de eso/ ¡ah!/ también noh quitaron loh zapatoh/ y na[d]a poh/ noh hicieron lo que hacen siempre/ eso de/ que te tiran mohtaza/ají/pehca[d]o/ to[d]a esa mierda/y dehpuéh tivimoh que salir a buhcar plata/ que eran cinco lucah// para que noh devolvieran la mochila y loh zapatoh" (H182, estrato medio alto).

Finalmente, en el ejemplo (4), nos encontramos frente a una narración que se construye mediante el patrón empobrecido, es decir, el entrevistado, de estrato medio alto, da a conocer los hechos de manera muy sucinta, sin detallar mayormente qué fue lo que ocurrió; de hecho, señala explícitamente "noh hicieron lo que hacen siempre". Luego, enumera una serie de actividades que, de acuerdo con sus planteamientos, son de conocimiento público, por lo que no detalla ni ordena los hechos de su historia tal como ocurrieron.

\section{CONCLUSIONES}

Los resultados más significativos que se desprenden del análisis anteriormente expuesto se pueden sintetizar como sigue:

1. A partir del análisis realizado a 64 narraciones de experiencias personales (32 de sujetos masculinos y 32 de representantes del habla femenina) de hablantes jóvenes de Santiago de Chile, que conformaron la muestra de 
nuestra investigación, podemos concluir que los informantes prefieren el patrón clásico para contar sus historias, es decir, la narración se va construyendo hasta un punto máximo, se refuerza evaluativamente en él y entonces se resuelve. De hecho, 42 narraciones se construyen con base en este patrón. Con una frecuencia bastante menor se registran el patrón de saltos múltiples (10 casos), el patrón empobrecido ( 7 casos), el patrón que termina en el punto máximo (4 casos) y el patrón desorientado ( 1 caso). Aunque la mayoría de las narraciones presenta una sucesión cronológica de acontecimientos, el patrón cronológico no se registró en nuestra muestra, porque las narraciones analizadas responden más bien a otro tipo de patrones y no a la mera descripción sucesiva de eventos. Tampoco se observó la presencia del patrón misceláneo, dado que todos nuestros relatos se ajustaron a alguno de los patrones anteriores.

2. Si observamos las diferencias de género dentro de cada patrón narrativo, podemos concluir que son las mujeres quienes con mayor frecuencia utilizan patrones que les permiten relatar de manera más "ordenada" los eventos acaecidos, basándose, sobre todo, en el patrón clásico; sin embargo, se trata de una diferencia mínima en comparación con los hablantes masculinos (34,6\% en el caso de las mujeres y $31,1 \%$ en los hombres). Asimismo, solo en el patrón que termina en el punto máximo se registra igual índice de frecuencia entre los representantes de ambos sexos ( 2 casos). El resto de los patrones registrados en nuestra muestra permite apreciar que las mujeres narran más fielmente la historia según los hechos acontecidos, aunque se trata, insistimos, de diferencias muy leves. Así, el patrón de saltos múltiples y el patrón empobrecido son mayoritariamente empleados por los hombres de nuestra muestra, lo que da cuenta de que no narran los hechos cronológicamente. Finalmente, el patrón clásico es más utilizado por las mujeres, lo que permite determinar que cuentan sus historias de un modo fidedigno.

3. Respecto del análisis que implicó la variable estrato social de los informantes, es posible concluir que existen algunas diferencias importantes de mencionar en cuanto a la frecuencia de empleo de los patrones narrativos en los cuatro estratos analizados: los estratos bajo y medio bajo presentan los índices más elevados de empleo del patrón clásico ( $22 \%$ en el estrato bajo y $17,1 \%$ en el estrato medio bajo), por lo tanto, concluimos que son los hablantes de los extremos inferiores de la escala socioeconómica quienes cuentan sus historias de manera más cercana a la realidad; esto es, respetando fielmente el orden en que acaecieron los hechos. Esta aseveración se puede comprobar al observar que en los estratos medio alto y medio, en general, se registraron las mayores frecuencias de empleo del patrón que termina en el punto máximo, del 
de saltos múltiples, del empobrecido y del desorientado, por lo que los relatos de los informantes de dichos estratos no narran sus historias tan fielmente, aunque, nuevamente, se trata de diferencias de frecuencias mínimas.

4. El hecho de que principalmente sean las mujeres de los estratos medio bajo y bajo de la comunidad en estudio quienes produzcan las narraciones más próximas a la realidad, esto es, relatadas siguiendo la estructura del patrón clásico, podría explicarse por la relativa mayor aproximación de los integrantes de dichos estratos al esquema narrativo característico de la "oralidad primaria". Sobre este punto debe precisarse que, originalmente, nuestra hipótesis era que las narraciones producidas por hablantes de ambos sexos de los estratos más bajos de la escala de estratificación socioeconómica producirían relatos incompletos en comparación con los pronunciados por representantes de los estratos medio alto y medio; sin embargo, los resultados de nuestro estudio dan cuenta de que a nivel socioeconómico no hay mayores diferencias en cuanto a la conformación de las narraciones, y que los representantes de los cuatro estratos en estudio generan relatos constituidos de manera lógica e, incluso, que al contrario de lo que pudiésemos pensar, en los estratos bajos las narraciones resultaron mejor relatadas que en los altos. Como ya señalamos, la explicación de este hallazgo podría encontrarse en la noción de oralidad primaria. El término "oralidad primaria", explicado por Havenlock (1995) hace referencia al tipo de lenguaje propio de una comunidad sin escritura. De esta forma, tal como señalan Prieto y San Martín (2002-2003), citando al sociólogo alemán Ferdinand Tönnies, el paso a la modernidad desde el orden tradicional implica un tránsito de Gemeinschaft -caracterizado por un tipo de relación social estrecha e íntima entre los individuos-a Gesellschaft-donde las relaciones sociales se caracterizan por la competencia, el interés propio y el progreso, entre otras características propias de la modernidad. Así, son los estratos socioeconómicos que se encuentran en el extremo superior de la escala social los que tienen más acceso a la cultura letrada, dejando de lado, en parte, la oralidad como recurso comunicativo; por lo tanto, en los estratos socioeconómicos bajos existe una mayor conciencia de la importancia de la cultura oral y al emplearla más, la desarrollan mejor. En consecuencia, no deja de ser importante dicho tránsito al intentar esbozar un ensayo explicativo de por qué en los estratos medio bajo y bajo las narraciones producidas por los hablantes que conforman nuestra muestra son tanto o más completas y lógicas que las producidas por los representantes del habla de los estratos medio y medio alto de la comunidad lingüística en estudio. 


\section{REFERENCIAS BIBLIOGRÁFICAS}

ACUÑA, ViRGinIA. 2006. La producción de segundas versiones más elaboradas de una historia en la conversación cotidiana. Oralia 9: 7-33.

Adam, Jean Michelle. 1999. Linguistique textuelle. Des genres de dicours aux textes. Paris: Nathan/HER.

Bassols, Margarida y Anna Torrent. 2003. Modelos textuales. Barcelona: Eumo- Octaedro.

Blas Arroyo, José Luis. 2005. Sociolingüística del español. Desarrollos y perspectivas en el estudio de la lengua española en contexto social. Madrid: Cátedra.

Calsamiglia, Helena y Amparo Tusón. 1999. Las cosas del decir. Manual de análisis del discurso. Barcelona: Ariel.

Chambers, Jack. 2003. Sociolinguistic theory. Londres: Blackwell

Charaudeau, Patrick y Dominique Maingueneau. 2005. Diccionario de análisis del discurso. Buenos Aires: Amorrortu editores.

Cheshire, Jenny. 2003. Sex and gender in variationist research. En Chambers, Jack, Peter Trudgill y Natalie Schilling-Estes (eds.). The handbook of language variation and change. Blackwell Publishing.

COATES, JeNnIFER. 2009 [1987]. Mujeres, hombres y lenguaje. Un acercamiento sociolingüistico a las diferencias de género. Fondo de Cultura Económica: México, D. F.

De Beaugrande, Robert. 2000. La saga del análisis del discurso. En Teun van Dijk (comp.). El discurso como estructura y proceso. Barcelona, Gedisa, pp. 67-106.

HAVENLOCK, ERIC. 1995. La ecuación oral-escrito: una fórmula para la mentalidad moderna. En Olson, David y Nancy Torrance (comps.). Cultura escrita y oralidad. Barcelona: Gedisa.

JiMÉNEZ, THEIRA. 2006. La narración infantil. Un estudio en niños de educación básica. Revista de investigación 60: 157-174. Universidad Central de Venezuela.

Jones, Deborah. 1980. Gossip: notes on women's oral culture. En C. Kramarae (ed.). The voices and words of women and men. Oxford: Pergamon Press.

Labov, William. 1972. The transformation of experience in narrative syntax. Language in the inner city. Studies in the Black English Vernacular. Filadelfia: University of Pennsylvania Press.

1992. Modelos Sociolingüisticos. Madrid: Cátedra.

Labov, William y Joshua Waletzky. 1967. "Narrative analysis". En June Helm (ed.). Essays on the verbal and visual arts. Seattle: University of Washington Press, pp. 12-44.

LaKoff, Robin. 1973. Language and woman's place. Language in Society 2: 45-79.

Lenski, Gerhardt. 1954. Status crystalization: A non-vertical dimension of social status. American Sociological Review 19: 405-413.

López Morales, Humberto. 2004. Sociolingüística. Madrid: Gredos.

Moreno Fernández, Francisco. 1998. Principios de sociolingüística y sociología del lenguaje. Barcelona: Ariel.

Prieto, Luis. 1995-1996. Análisis sociolingüístico del dequeísmo en el habla de Santiago de Chile. Boletín de Filología de la Universidad de Chile XXXV: 379-452.

Prieto, Luis y Abelardo San Martín. 2002-2003. Diferencias de género en el empleo del discurso referido: aproximación sociolingüística y pragmático-discursiva. Boletín de Filología de la Universidad de Chile XXXIX: 269:304.

Reyes, Claudia. 1996. Estrategias narrativas en la zona metropolitana de Monterrey. En Rodríguez Flores, Dora y Lidia Rodríguez Alfano (eds.). Lenguaje y sociedad: Metodología y análisis aplicados a El habla de Monterrey. México: Trillas, pp. 101-120. 
Shiro, Martha. 2007. El discurso narrativo oral en la vida cotidiana: géneros y procesos. En Bolívar, Adriana (ed.). Análisis del discurso. Caracas: Colección Minerva, Manuales Universitarios, pp. 121-143.

Soler, Sandra. 2004. Discurso y género en historias de vida. Una investigación de relatos de hombres y mujeres en Bogotá. Publicaciones del Instituto Caro y Cuervo: Bogotá.

TANNEN, DeBorah. 1990a. La comunicación entre hombres y mujeres a la hora del trabajo. Bueno Aires-Argentina: Javier Vergara Editor S. A.

1990b. You just don't understand. Women and man in conversation. Nueva York: Ballantine Books.

1996: Género y discurso. Barcelona: Paidós.

van DiJK, Teun, 2000. "Superestructuras". En La ciencia del texto. Barcelona/Buenos Aires: Paidós.

2005. Estructuras y funciones del discurso. Una introducción interdisciplinaria a la lingüística del texto y a los estudios del discurso. Madrid: Siglo veintiuno editores. Werlich, Egon. 1975. Typologie der Texte. Munich: Fink. 